\title{
Four Years of Real-Time GRB Followup by BOOTES-1B (2005-2008)
}

\author{
Martin Jelínek, ${ }^{1}$ Alberto J. Castro-Tirado, ${ }^{1}$ Antonio de Ugarte Postigo, ${ }^{2}$ Petr Kubánek, ${ }^{1,3}$ \\ Sergey Guziy, ${ }^{1}$ Javier Gorosabel, ${ }^{1}$ Ronan Cunniffe, ${ }^{1}$ Stanislav Vítek, ${ }^{4}$ René Hudec, ${ }^{4,5}$ \\ Victor Reglero, ${ }^{3}$ and Lola Sabau-Graziati ${ }^{6}$
}

${ }^{1}$ Instituto de Astrofísica de Andalucía (IAA CSIC), 18008 Granada, Spain

${ }^{2}$ Instituto Nazionale di Astrofisica, 23807 Milano, Italy

${ }^{3}$ Image Processing Laboratory, Universitat de Valencia, 46980 Valencia, Spain

${ }^{4}$ Fakulta Elektrotechnická, ĆVUT, 16627 Praha, Czech Republic

${ }^{5}$ Astronomický ústav Akademie vĕd (ASÚ AV ČR), 25165 Ondřejov, Czech Republic

${ }^{6}$ Instituto Nacional de Técnica Aeroespacial, 28850 Torrejón de Ardoz, Spain

Correspondence should be addressed to Martin Jelínek, mates@iaa.es

Received 25 July 2009; Revised 18 November 2009; Accepted 12 January 2010

Academic Editor: Joshua S. Bloom

Copyright (c) 2010 Martin Jelínek et al. This is an open access article distributed under the Creative Commons Attribution License, which permits unrestricted use, distribution, and reproduction in any medium, provided the original work is properly cited.

\begin{abstract}
Four years of BOOTES-1B GRB follow-up history are summarised for the first time in the form of a table. The successfully followed events are described case by case. Further, the data are used to show the GRB trigger rate in Spain on a per-year basis, resulting in an estimate of 18 triggers and about 51 hours of telescope time per year for real-time triggers. These numbers grow to about 22 triggers and 77 hours per year if we include also the GRBs observable within 2 hours after the trigger.
\end{abstract}

\section{Introduction}

BOOTES-1B (see also $[1,2]$ ) is an independent robotic observatory with a $30 \mathrm{~cm}$ aperture telescope dedicated primarily to followup of gamma-ray burst (GRBs). Since 2003, it has used RTS2 [3] as an observing system. It is located at the atmospheric sounding station (Estación de Sondeos Atmosféricos-ESAt, INTA) of El Arenosillo in Andaluca, Spain (at lat: $37^{\circ} 06^{\prime} 16^{\prime \prime} \mathrm{N}$, long: $06^{\circ} 43^{\prime} 58^{\prime \prime} \mathrm{W}$ ). A nearby, older dome (BOOTES-1A) is used for complementary wider angle instruments (Figure 2).

We present results of our GRB follow-up programme. In a large table, we show a 4-year long follow-up log of BOOTES-1B GRBs-including all triggers available in real time which were or should have been received and processed by the system. This selected sample of GRBs is then used to provide a basic idea of how much time is needed at the telescope to observe GRB optical afterglows.

\section{Robotic Telescope Configuration}

The telescope is built mostly from commercially available components-a Paramount ME from Software Bisque and a $D=30 \mathrm{~cm}$ Schmidt-Cassegrain optical tube assembly from Meade. Over time, four distinct system configurations were used, including also two 8-inch S-C telescopes (Figure 1).

2.1. Original Meade-Stereoscopic System. The original BOOTES project idea of a new generation of robotic telescopes was very simple, BOOTES-1B would-simultaneous with an identical setup at BOOTES-2 for parallax abilitylook for optical transients in an extended area of the sky with wide field cameras. Both systems would use a commercial 12-inch Meade LX-200 "robot." The wide-field cameras were considered a primary instrument, while the ability to followup with a large telescope was an option. Between 1998 and 2002 the wide-field system provided simultaneous 


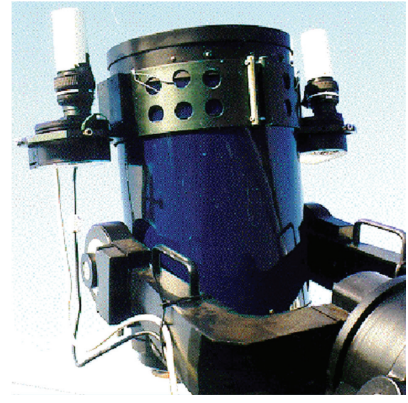

(a)

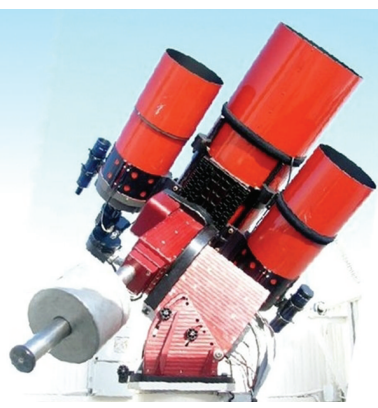

(c)

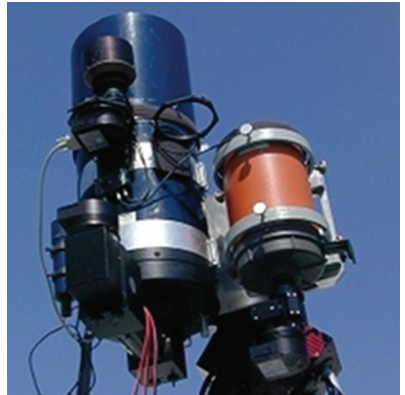

(b)

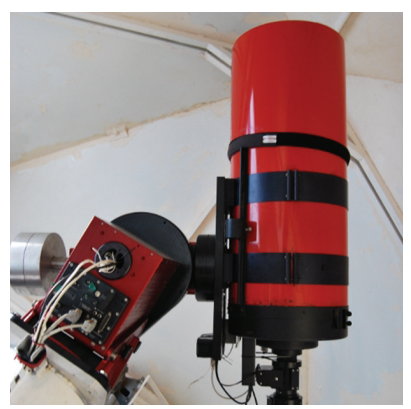

(d)
FIgURE 1: Four historic BOOTES-1B configurations.

limits for several CGRO/BATSE and BeppoSAX GRBs, most notably the candidate afterglow for the short GRB 000313 [4]. The $30 \mathrm{~cm}$ telescope was successfully used to followup GRB 030329.

Although we made the original system able to observe, it kept having problems. It required an operator presence several times per week and, despite a notable effort, the fork mount's electronics had to be exchanged several times. Because of that, we decided to purchase another mount.

\subsection{The Prototype. The new incarnation of BOOTES-1B} was in preparation since mid-2002, and the first prototype was put together in November 2002 in order to followup INTEGRAL bursts. The most important change was the mount to be used-the Paramount ME from Software Bisque. The system was still carrying a wide field camera, but a shift had been made in priorities - the wide field camera performed monitoring of satellite field of view and the telescope pointed when a trigger was received. The early stage was, however, plagued with technical and organizational problems which eventually delayed the first real-time realGRB follow-up until early 2005.

The prototype carried three instruments on a large aluminium base plate: the $30 \mathrm{~cm}$ telescope with a field spectrograph [5] plus an SBIG-ST8 camera, the $20 \mathrm{~cm}$ Meade (originally BOOTES-1A) telescope with an SBIG-ST9 camera observing in a fixed V-band filter, and an unfiltered wide-field $18 \mathrm{~mm} / 1: 2.8$ with an SBIG ST-8 CCD $\left(43^{\circ} \times 29^{\circ}\right)$.

BOOTES-1B was operating with this setup for about a year-on June 2004, it was dismounted and sent to the Workshop in Ondřejov for a definitive solution.

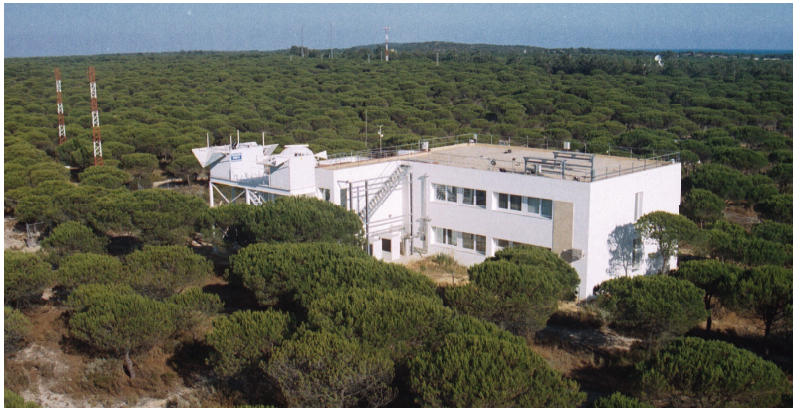

FIgure 2: The building of ESAt with domes of BOOTES-1A and BOOTES-1B (2002).

2.3. Triple Telescope. The prototype was very heavy and from the beginning had some problems. In September 2004 BOOTES-1B finally received an upgrade-together with the $30 \mathrm{~cm}$ telescope, there were also two $20 \mathrm{~cm}$ telescopes( One of them lent personally by AJCT) for direct imaging in different filters. The system was completely redesigned with many mechanical improvements and was built to be as light as possible to allow the mount working at its maximum slewing speed. In belief that the rapid dissemination and fast followup after the launch of Swift would lead into relatively frequent detections of bright optical counterparts, the $30 \mathrm{~cm}$ telescope was equipped with a field spectrograph and two $20 \mathrm{~cm}$ telescopes with fixed $V \& I$-band filters. The limiting magnitude of all three instruments was $V \sim 16$ for a 60 second exposure. The wide-field cameras were moved from BOOTES-1B to BOOTES-1A.

Later, during the telescope operation, it became clear that the GRB optical transients were not as bright as had been expected and so the spectrograph on the $30 \mathrm{~cm}$ telescope was replaced with a direct imaging CCD with Rband filter-improving the limiting magnitude but losing the spectroscopic ability.

On April 23, 2006, The ESAt building was struck by lightning during a storm, destroying a major part of BOOTES-1B electronics. It took more than a year to get BOOTES-1B definitively back online.

2.4. Single $30 \mathrm{~cm}$ Telescope. During the lengthy reconstruction of BOOTES-1B, the follow-up strategy was reconsidered: in the interest of detecting more optical transients, the filter(s) were abandoned $(\sim 2.5 \times$ or 1 magnitude gain in sensitivity). The limiting magnitude of an unfiltered $120 \mathrm{~s}$ exposure would be about 18.0-effectively doubling the likelihood of getting an optical transient in comparison with the R-band imaging (cf. Figure 4). Both $20 \mathrm{~cm}$ telescopes were dropped because of lack of suitable CCD cameras available for them. Since then, BOOTES-1B has only a single $30 \mathrm{~cm}$ telescope.

Any observations obtained after June 15, 2007 have been obtained without filter ( $W$ for white). We calibrate them against $R$-band, which, in the case of no color evolution of the optical counterpart, is expected to result in a small constant offset in magnitude. 


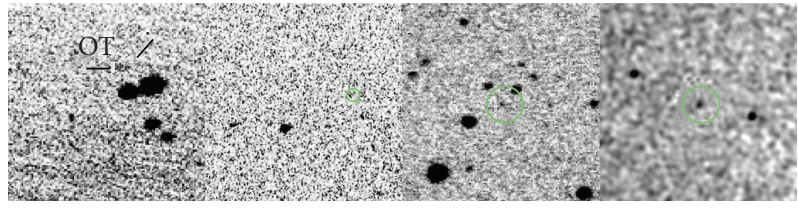

(a)

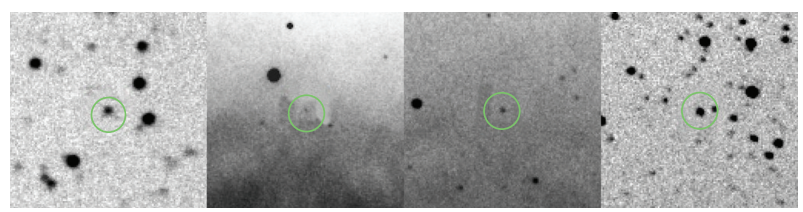

(b)

FIGURE 3: GRB optical transient detections by BOOTES-1B: (a) GRB 050824, GRB 050922C, GRB 051109A, GRB 080330. (b) GRB 080413B, GRB 080430, GRB 080602B, GRB 080605.

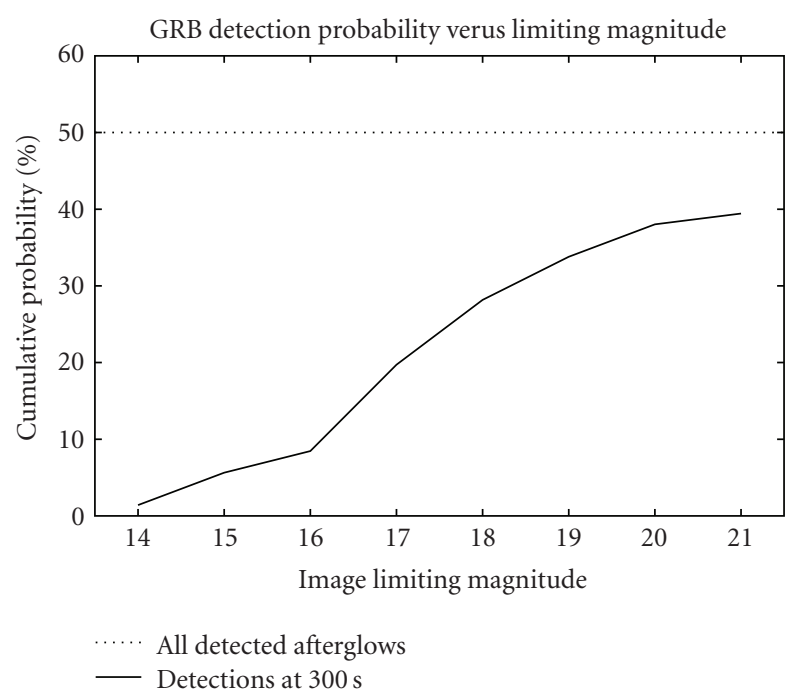

Figure 4: The graph (based on $T_{300}$ data from Table 3) showing the likelihood of detection of an optical afterglow of a GRB as a function of the magnitude the telescope can detect (in the time interval discussed here). The dotted line delimits 50\%-the ratio of GRBs in our data for which there was eventually discovered an optical transient.

\section{Real-Time GRB Followup}

BOOTES-1B could have received during the past 4 years (since January 2005 until December 2008) 86 GRB triggers via GCN, which could have been followed in real time or would become observable within the following two hours. Table 3 summarizes these triggers, noting, among BOOTES$1 \mathrm{~B}$ status of the followup, also the brightness of the GRB optical counterpart if it is known. The magnitude estimation search was done with a heavy use of GRBlog [6]. Successful followups are summarized in Table 1, the detected optical transients also in Figure 3. We use these data to construct a "limiting magnitude versus likelihood of detection" graph (Figure 4).

\subsection{GRB Triggers Followed by BOOTES-1B}

(a) GRB 050215B. GRB was discovered by Swift/BAT at 02:33:43.2 UT. BOOTES received the notice, but because of a software error waited with the slew until $\sim 22$ minutes after the GRB. We coadded $600 \mathrm{~s}$ exposures taken by both $20 \mathrm{~cm}$ telescopes to obtain limits of $V>16.5$ and $I>15.0$ [7].

(b) GRB 050505. First image of this Swift-discovered GRB [8] was obtained at 23:32:30 UT, that is, $609 \mathrm{~s}$ after the trigger and $70 \mathrm{~s}$ after receiving the coordinates. No optical afterglow was detected [9].

(c) GRB 050509A. At the time of this trigger, the dome was still operating independently on the rest of the system; we obtained the first image $23 \mathrm{~s}$ after the GRB trigger ( $6 \mathrm{~s}$ after receiving the alert). The dome was closed due to what we consider a false trigger on the rain sensor. The first useful $10 \mathrm{~s}$ exposure was obtained 63.8 minutes after the burst and has a limiting magnitude of $V>14.9$. A coadd of first $112 \times 10 \mathrm{~s}$ exposures with an exposure mean time of 88.0 minutes after the GRB has a limit of $V>18.1$.

(d) GRB 050509B. This was a Swift-detected short gammaray burst [10]. Starting $62 \mathrm{~s}$ after the trigger, BOOTES-1B seems to have obtained the world-first data set of this short duration GRB. However, bad luck caused that the location of the GRB on the sky coincided with the tip of a nearby antenna and its signalling light. The limiting magnitude is thus seriously degraded. The first 10-second exposure has a limiting magnitude of $V>11.5$; a combination of the first $12 \times 10 \mathrm{~s}$ exposures provides $V>12.5$.

(e) GRB 050525A. GRB 050525A [11] was the first BOOTES$1 \mathrm{~B}$ burst for which a detection was obtained. The telescope started the first exposure $383 \mathrm{~s}$ after the GRB trigger $(28 \mathrm{~s}$ after receiving the notice). An optical afterglow with $V \simeq$ 15.0 was detected.

(f) GRB 050528. We observed the errorbox [12] at 04:07:56 UT, that is, starting $71 \mathrm{~s}$ after the burst and $28 \mathrm{~s}$ after receiving the trigger in a light twilight, setting the limit to the possible GRB counterpart to $V>13.8$ and $I>13.0$ during the first $60 \mathrm{~s}$ after the beginning of our observation [13].

(g) GRB 050730. Located at 19:58:23 UT, this GRB was very low above horizon in real time; BOOTES-1B obtained few exposures starting $233.4 \mathrm{~s}$ after the GRB, when the system failed. The images did not provide detection of the $17.0 \mathrm{mag}$ optical transient discovered by both Swift/UVOT [14] and $1.5 \mathrm{~m}$ telescope at OSN [15].

(h) GRB 050805B. This short burst was localized by Swift at 20:41:26 UT; BOOTES obtained first images $62.2 \mathrm{~s}$ after the trigger ( $7.2 \mathrm{~s}$ after receiving the trigger). No optical transient was detected. The first 10 -second exposure has a limiting magnitude of $R>16$, a combination of first five images 
(exposure mean time $118 \mathrm{~s}$ after the GRB) has a limit of $R>17.0$.

(i) GRB 050824. The optical afterglow of this GRB was discovered with the $1.5 \mathrm{~m}$ telescope at Sierra Nevada [16, 17]. BOOTES-1B was, however, the first telescope to observe this optical transient, starting $636 \mathrm{~s}$ after the trigger with $R \simeq 17.5$. The weather was not stable and the focus not perfect, but BOOTES-1B worked as expected. Eventually, several hours of data were obtained.

(j) GRB050904. BOOTES-1B reacted to this GRB, starting $124 \mathrm{~s}$ after the trigger. There was a hot pixel close to the GRB location, which made us believe we might have a detection in the $R$-band, which was issued in the first BOOTES-1B circular. Later, the observation revised as a limit $(R>18.2)$ which was used to compute the record redshift of this GRB [18]. The $I$-band camera of the $20 \mathrm{~cm}$ telescope, unluckily, failed.

(k) GRB 050922C. This bright burst was detected by Swift /BAT at 19:55:50 UT. BOOTES was not very lucky; the weather on the station was bad. Instead of a limiting magnitude of $\sim 17.0$ for a 30 -second exposure, we got 12.9. The afterglow was eventually detected with the $R$-band camera (at the $30 \mathrm{~cm}$ telescope) on few occasions between flying clouds. The first weak detection was obtained $228 \mathrm{~s}$ after the GRB trigger gave $R \simeq 14.5$.

(l) GRB 051109A. This is the only GRB ever detected by BOOTES-1B simultaneously in more than one filter. The first images were obtained $54.8 \mathrm{~s}$ after the burst in $R$ and $I$ bands [19].

(m) GRB 051211B. Observation of this burst started $42 \mathrm{~s}$ after the burst. A 30-second R-band exposure was obtained, but the camera failed after getting this image. Only useless defocused $I$-band images were taken with the $20 \mathrm{~cm}$ telescope [20].

(n) GRB 051221B. This GRB was detected by Swift/BAT at 20:06:48 UT [21]. BOOTES-1B slewed to the position and started obtaining images $27.8 \mathrm{~s}$ after receiving the alert (234.8 s after the burst). We did not find any new source in our images [22]. 30 minutes after the trigger, a faint 21 magnitude afterglow was discovered elsewhere [23].

(o) GRB 060421. BOOTES-1B reacted to this GRB within $61 \mathrm{~s}$ after the trigger. Images were not of a great quality, yielding a limit of $R>14$ for the first 10-second exposure and $R>16$ for the combination of 30 images (exposure mean time was $547 \mathrm{~s}$ after the trigger).

(p) GRB 061110B. The GRB was detected by Swift/BAT at $T_{0}=21: 58: 45 \mathrm{UT}$, but the notices were delayed by $626 \mathrm{~s}$ because Swift was performing downlink. BOOTES-1B started to slew immediately after reception of the trigger, obtaining the first image $698 \mathrm{~s}$ after the burst $(72 \mathrm{~s}$ after the GCN notice). When seven 60-second exposures were obtained, a communication error with the mount occurred. The communication was later restored and further 19 images were obtained starting 22:49:49 UT (0.85 hour after burst). Last image was obtained at 23:36:20 (1.62 hours after burst).

Combination of the first 7 images (limiting magnitude $\sim 17.2$ mag each) with the exposure mean time of $938 \mathrm{~s}$ after the GRB trigger was found to have a magnitude limit of 18.0. Combination of 11 images obtained between 22:49:49 UT and 23:03:45 UT (lim 16.9 mag each) yields a limit of $\sim 18.2$ with mean time $T_{0}+3452 \mathrm{~s}$.

(q) GRB 071101. In the process of reconstruction, the $30 \mathrm{~cm}$ telescope from BOOTES-1B was re-installed at the BOOTES2 site in La Mayora (Málaga). This GRB was the first event successfully observed by the repaired telescope at this site. The GRB trigger [24] was at 17:53:46 UT. BOOTES started imaging $54.8 \mathrm{~s}$ after the burst $(23.3 \mathrm{~s}$ after receiving the coordinates). No afterglow was detected, an unfiltered, $R$ band calibrated limit of $W>17.0$ was estimated [25].

(r) GRB 071109. INTEGRAL detected this GRB at 20:36:05 UT [26]. BOOTES followed up $58.5 \mathrm{~s}$ after the GRB (30.9s after receiving the alert). Because of high altitude clouds, the telescope performance was reduced, yielding an unfiltered limit of $\sim 13.0$ in the first $10 \mathrm{~s}$ exposure [27].

(s) GRB 080330. This GRB [28] happened during the first day of recomissioning of BOOTES-1B after its move from BOOTES-2 site in La Mayora. The GCN client was not yet operational and at the time of the GRB we were focusing the telescope. First image was obtained $379 \mathrm{~s}$ after the GRB trigger and the optical afterglow was detected with magnitude $\sim 16.3$ on the first image. A bug in the centering algorithm caused a loss of part subsequent data. Further detections were obtained starting 21 minutes after the GRB when the problem was fixed.

( $t$ ) GRB 080413A. BOOTES-1B started obtaining images of the GRB 080413A [29] starting $60.7 \mathrm{~s}$ after the trigger $(46.3 \mathrm{~s}$ after reception of the alert). $\mathrm{A} W \simeq 13.3$ magnitude optical afterglow was found [30].

(u) GRB 080430. BOOTES-1B obtained the first image of this GRB [31] $34.4 \mathrm{~s}$ after the trigger. An optical transient was found with a magnitude of $W \simeq 15.5$ [32].

(v) GRB 080603B. This GRB happened at BOOTES-1 site during sunset. We obtained first useful images starting one hour after the trigger. An $W \simeq 17.4$ optical transient was found.

(w) GRB 080605. GRB 080605 was observed starting 41.9s after the trigger. A rapidly decaying optical afterglow with $W \simeq 14.8$ was found [33]. 
(x) GRB 081003B. INTEGRAL detected this GRB at 20:36:05 UT [34]. BOOTES started obtaining unfiltered images at 20:48:49 UT (41 s after the GRB trigger and $17.4 \mathrm{~s}$ after the GCN notice); single images have a detection limit of $W>$ 14 mag. The combination of the first 32 images with an exposure mean time of $80 \mathrm{~s}$ after the GRB has a limit of $W>$ 17.6 mag (calibrated against GSC2). Neither shows any new sources within the GRB errorbox [35].

\section{Implications}

4.1. Success Rate. Of the 89 triggers, 45 were processed in realtime and observed if possible; in 44 cases the system could not respond. This makes the overall failure rate quite high (50\%). 29 triggers were, however, lost due to longterm failures resulting from the telescope being struck by lightning. 8 more triggers failed during the first 6 months of operation, when the system was not yet fully stable and one was lost during maintenance (and followed manually). 6 triggers out of $47(13 \%)$ were lost unexpectedly during the 963 nights of telescope operation if we do not count the first semester of 2005.

4.2. Planning. When specifying the GRB follow-up needs, the number of nights (hours) spent observing GRBs has to be estimated. Under various follow-up strategies, we may derive different results.

Due to various instrumental effects (like a passage through the South Atlantic Anomaly) related to the satellite Swift, an offset from the overall triggering statistic which would depend on a geographical location could be found.

In Table 3, the fourth column has the time in hours until the first set of the event location below $10^{\circ}$ of altitude or until the Sun rises above $-15^{\circ}$ of altitude. For nonrealtime events, this is the time the location spends on the night sky; for real-time triggers, it is the time between the trigger and the moment when the target becomes unobservable.

For a small telescope, we assume that once the GRB is real-time triggered, it is unlikely to detect it the following night (i.e., after $\sim 24$ hours); so we assume the following simple follow-up strategy: Let the telescope observe the GRB once it becomes accessible for the first time (which is immediately for real-time triggers) and let it observe until the GRB sets or the night ends. Do not observe any further nights. Under the given assumptions, we get the following observing needs (assuming perfect weather).

(y) Real-time triggers. There have been 72 real-time triggers during the studied 4 years; during their first nights they accumulated 202 hours.

So if we allow only real-time followable triggers to be observed, we would need $\sim 18$ triggers per year (once per 20 days) and on average 2.8 hours (max. 8.0 hours) of observing time per trigger, 50.5 hours per year. Such a program would consume about $2 \%$ of the telescope time.

(z) Extended set. In the extended set, we assume that GRBs that would become observable within 2 hours after the event
TABLE 1: Summary of GRBs successfully followed by BOOTES-1B.

\begin{tabular}{|c|c|c|c|}
\hline GRB & $T_{\text {obs }}-T_{\text {trigger }}$ & $T_{\text {obs }}-T_{\text {notice }}$ & mag \\
\hline 050215B & $22 \mathrm{~min}$ & & $V>16.5, I>15.0$ \\
\hline 050505 & $609 \mathrm{~s}$ & $70 \mathrm{~s}$ & $V>14.0$ \\
\hline 050509A & $63.8 \mathrm{~min}$ & & $V>18.1$ \\
\hline 050509B & $62 s$ & $48 \mathrm{~s}$ & $V>12.5$ \\
\hline 050525A & $383 \mathrm{~s}$ & $28 \mathrm{~s}$ & $V \simeq 15.0$ \\
\hline 050528 & $71 \mathrm{~s}$ & $28 \mathrm{~s}$ & $V>13.8, I>13.0$ \\
\hline 050730 & $233 \mathrm{~s}$ & $172 \mathrm{~s}$ & $R>16$ \\
\hline 050824 & $636 s$ & $55.8 \mathrm{~s}$ & $R \simeq 17.5$ \\
\hline 050805B & $62 \mathrm{~s}$ & $17 \mathrm{~s}$ & $R>16.0$ \\
\hline 050904 & $124 \mathrm{~s}$ & $43 \mathrm{~s}$ & $R>18.2$ \\
\hline 050922C & $228 \mathrm{~s}$ & $62.3 \mathrm{~s}$ & $R \simeq 14.5$ \\
\hline 051109A & $54.8 \mathrm{~s}$ & $27 \mathrm{~s}$ & $R \simeq 16.2$ \\
\hline 051211B & $42 \mathrm{~s}$ & $48.4 \mathrm{~s}$ & $I>14.0$ \\
\hline 051221B & $234.8 \mathrm{~s}$ & $27.8 \mathrm{~s}$ & $V>13.3$ \\
\hline 060421 & $61.2 \mathrm{~s}$ & $47.6 \mathrm{~s}$ & $R>16.0$ \\
\hline 061110B & $698 \mathrm{~s}$ & $72 \mathrm{~s}$ & $R>18.0$ \\
\hline 071101 & $54.8 \mathrm{~s}$ & $23.3 \mathrm{~s}$ & $W>17.0$ \\
\hline 071109 & $58.5 \mathrm{~s}$ & $30.9 \mathrm{~s}$ & $W>13.0$ \\
\hline 080330 & $379 s$ & & $W \simeq 16.3$ \\
\hline 080413A & $60.7 \mathrm{~s}$ & $46.3 \mathrm{~s}$ & $W \simeq 13.3$ \\
\hline 080430 & $34.4 \mathrm{~s}$ & $22.1 \mathrm{~s}$ & $W \simeq 15.5$ \\
\hline 080603B & $60 \mathrm{~min}$ & & $W \simeq 17.4$ \\
\hline 080605 & $41.9 \mathrm{~s}$ & $29.3 \mathrm{~s}$ & $W \simeq 14.8$ \\
\hline 081003 & $41 \mathrm{~s}$ & $17.4 \mathrm{~s}$ & $W>14.8$ \\
\hline
\end{tabular}

TABLE 2: Results of the GRB-planning statistic.

\begin{tabular}{lcc}
\hline & Real time only & Up to 2 hours \\
\hline triggers/year & 18 & 22 \\
hours/year & 50.5 & 78.5 \\
hours/trigger & 2.8 & 3.5 \\
days/trigger & 20.3 & 16.6 \\
\hline
\end{tabular}

would also be followed. We would need $\sim 22$ triggers per year, each with an average length of 3.5 hours. In total we would need 78.5 hours per year, or about $3 \%$ of the telescope time. Summary of the triggering statistic cf. Table 2.

4.3. Optical Afterglow Brightnesses. As a representative value of GRB optical transient brightness, important for real-time followup, we have chosen its magnitude at $300 \mathrm{~s}$ after the trigger. It turns out that it is not easy to find a uniform sample, and available magnitudes and limits are a mixture of different passbands, mainly $V, R$ and unfiltered CCD magnitudes. For a general idea of how bright an OT could be this is, however, good enough. Figure 4 shows a cumulative probability of detecting an OT five minutes after the trigger with a telescope is able to detect a given magnitude. For many GRBs, the brightness at this early time is unknown, or only a limit from small telescopes has been established, so this curve is actually a slight underestimation. 
TABLE 3: The Great Table of BOOTES-1B GRBs. "Target" is the RTS2 target number at BOOTES-1B. $t_{1}$ is the time delay between the GRB trigger and the possible start of observation. $t_{\mathrm{obs}}$ is the amount of time for which the GRB can be followed until it sets for the first time. $m_{300}$ is the known brightness of the GRB optical transient $300 \mathrm{~s}$ after the event. $d T$ is the delay of BOOTES-1B followup.

\begin{tabular}{|c|c|c|c|c|c|c|}
\hline Object & Target & $t_{1}[\mathrm{~h}]$ & $t_{\mathrm{obs}}[\mathrm{h}]$ & $m_{300}[\mathrm{mag}]$ & $d T$ & observation status \\
\hline 050128 & - & +0.0 & 1.8 & - & - & No link to GCN \\
\hline 050208 & - & +0.0 & 4.2 & - & - & No link to GCN \\
\hline 050215B & 5064 & +0.4 & 10.0 & $\gtrsim 16^{\dagger}[36]$ & $22 \mathrm{~m}$ & $V, I$ limits [7] \\
\hline 050306 & 5075 & +0.2 & 1.8 & $>16[37]$ & $86 s$ & w/roof closed \\
\hline 050416B & 5109 & +0.0 & 2.8 & - & - & grbd failure \\
\hline 050421 & 5112 & +0.0 & 0.2 & $>18.4[38]$ & - & hw problems \\
\hline 050502A & - & +0.0 & 1.8 & $16.3[39]$ & - & grbd failure \\
\hline 050505 & 5123 & +0.0 & 2.2 & $-^{\dagger}$ & $609 \mathrm{~s}$ & clouds, $V, I$ limits \\
\hline 050509A & 5129 & +0.0 & 2.2 & $>18.2[40]$ & $23 \mathrm{~s}$ & hw problems, later limit \\
\hline 050509B & 5130 & +0.0 & 0.0 & $>20.8[41]$ & $62 \mathrm{~s}$ & OK, $V>12.5$, antenna hit! \\
\hline 050520 & - & +0.0 & 3.6 & $>16.6$ & - & GCN connection lost \\
\hline 050525A & 5136 & +0.0 & 3.6 & $14.7[42]$ & $383 \mathrm{~s}$ & OK, V-band lightcurve \\
\hline $050714 \mathrm{~A}$ & 1037 & +0.0 & 3.6 & - $^{\dagger}$ & $10 \mathrm{~m}$ & manually, later limit \\
\hline 050730 & 50008 & +1.2 & 1.6 & $17.4[14]$ & $1 \mathrm{~h} 40 \mathrm{~m}$ & limits \\
\hline 050805B & 50015 & +0.2 & 7.2 & - & $62 \mathrm{~s}$ & Limits \\
\hline 050824 & 50032 & +0.0 & 5.2 & $17.5[17]$ & $636 s$ & detection [17] \\
\hline 050904 & 50055 & +0.0 & 2.8 & $-^{\dagger}$ & $124 \mathrm{~s}$ & R-band limit [18] \\
\hline 050922C & 50090 & +0.0 & 6.2 & $15.5[43]$ & $228 \mathrm{~s}$ & detections between clouds \\
\hline 051109A & 50126 & +0.0 & 1.4 & 16.8 & $54.8 \mathrm{~s}$ & detection in R,I \\
\hline 051111 & - & +0.0 & 4.6 & $14.9[44]$ & - & GCN connection lost \\
\hline 051211A & 50144 & +0.0 & 3.2 & $\underline{-}^{\dagger}$ & - & CCD failure \\
\hline 051211B & 50146 & +0.0 & 4.8 & $>14.0$ & $50 \mathrm{~s}$ & OK, limits [20] \\
\hline 051221B & 50151 & +0.0 & 3.8 & $>18.2[45]$ & $235 \mathrm{~s}$ & OK, limits [22] \\
\hline 051227 & 50155 & +1.6 & 10.6 & $>19.2[46]$ & $59 \mathrm{~m}$ & bad weather \\
\hline 060111A & 50162 & +0.0 & 2.0 & $>18.3[47]$ & $296 \mathrm{~s}$ & during maintenance, limit \\
\hline 060121 & - & +0.0 & 7.8 & $-^{\dagger}$ & - & telescope OFF \\
\hline 060123 & 50171 & +0.0 & 7.6 & - & - & bad weather \\
\hline 060130 & 50173 & +0.0 & 1.2 & - & - & bad weather \\
\hline 060203 & - & +0.0 & 6.2 & - & - & bad weather+no GCN \\
\hline 060204C & - & +0.0 & 9.6 & $>18.7[48]$ & - & bad weather+no GCN \\
\hline 060206 & - & +0.0 & 1.4 & $16.5[49]$ & - & GCN connection lost \\
\hline 060219 & 50185 & +1.0 & 6.0 & $>18.6[50]$ & - & bad weather \\
\hline 060319 & 50190 & +0.0 & 4.4 & $>19^{\dagger}[51]$ & - & bad weather \\
\hline 060418 & 50207 & +0.0 & 1.4 & $14.2[52]$ & - & bad weather \\
\hline 060421 & 50208 & +0.0 & 3.8 & $>16.8[53]$ & $61.2 \mathrm{~s}$ & Limit \\
\hline 060424 & - & +0.0 & 0.0 & $-^{\dagger}$ & - & hw failure \\
\hline 060502A & - & +0.0 & 1.0 & $18.7[54]$ & - & hw failure \\
\hline 060507 & - & +0.0 & 2.0 & $>15.5^{\dagger}[55]$ & - & hw failure \\
\hline 060512 & - & +0.0 & 4.6 & $17.15[56]$ & - & hw failure \\
\hline 060515 & - & +0.0 & 1.4 & $>16.2[57]$ & - & hw failure \\
\hline 060522 & - & +0.0 & 1.6 & $19.65[58]$ & - & hw failure \\
\hline 060602A & - & +0.0 & 1.2 & $>15^{\dagger}[59]$ & - & hw failure \\
\hline 060602B & - & +0.0 & 3.6 & - & - & hw failure \\
\hline 060712 & - & +0.2 & 3.0 & $>14.5[60]$ & - & hw failure \\
\hline 060814 & - & +0.0 & 0.8 & $>17.4[61]$ & - & hw failure \\
\hline 060825 & - & +0.0 & 1.4 & $>18.3[62]$ & - & hw failure \\
\hline 060901 & - & +1.6 & 5.2 & $\ldots^{\dagger}$ & - & hw failure \\
\hline 060904A & - & +0.0 & 0.2 & $>19.5[63]$ & - & hw failure \\
\hline 060904B & - & +0.0 & 2.2 & $\sim 17[64,65]$ & - & hw failure \\
\hline
\end{tabular}


TABle 3: Continued.

\begin{tabular}{|c|c|c|c|c|c|c|}
\hline Object & Target & $t_{1}[\mathrm{~h}]$ & $t_{\mathrm{obs}}[\mathrm{h}]$ & $m_{300}[\mathrm{mag}]$ & $d T$ & observation status \\
\hline 060929 & 50212 & +0.0 & 1.2 & $>17.0[66]$ & - & bad weather \\
\hline 061019 & 50220 & +0.0 & 1.0 & $>14.8^{\dagger}[67]$ & - & bad weather \\
\hline 061110B & 50228 & +0.0 & 2.2 & $>17.8^{\dagger}[68]$ & $11 \mathrm{~m} 38 \mathrm{~s}$ & $>18.1 \mathrm{OK}$ \\
\hline 061217 & 50240 & +0.0 & 2.6 & $>19.2[69]$ & - & mount failure \\
\hline 061218 & 50242 & +0.0 & 2.0 & $>18.6[70]$ & - & mount failure \\
\hline 061222B & 50245 & +0.0 & 0.6 & $18.0[71]$ & - & mount failure \\
\hline 070103 & 50246 & +0.0 & 2.8 & $>19.6[72]$ & - & mount failure \\
\hline 070129 & 50253 & +0.0 & 0.4 & $>19.2^{\dagger}[73]$ & - & mount failure \\
\hline 070219 & - & +0.0 & 4.8 & $>20.0[74]$ & - & mount failure \\
\hline 070220 & 50258 & +0.0 & 1.2 & $>19.6[75]$ & - & mount failure \\
\hline 070223 & 50259 & +0.0 & 4.6 & $>21.4^{\dagger}[76]$ & - & mount failure \\
\hline 070224 & 50260 & +1.4 & 8.0 & $>20.1^{\dagger}[77]$ & - & mount failure \\
\hline 070406 & 50277 & +0.0 & 4.0 & - & - & mount failure \\
\hline 070411 & - & +0.0 & 3.2 & $\sim 18.3[78]$ & - & mount failure \\
\hline 070412 & - & +0.0 & 3.2 & $>20.7$ [79] & - & mount failure \\
\hline 070429A & 50286 & +1.6 & 1.0 & $>18.0[80]$ & - & mount failure \\
\hline 070531 & - & +0.0 & 1.4 & $>19.9[81]$ & - & mount failure \\
\hline 070610 & - & +0.4 & 6.2 & $\sim 19[82]$ & - & mount failure \\
\hline 070704 & - & +1.4 & 6.2 & $>21.2[83]$ & - & manually disabled \\
\hline 070714A & 50010 & +0.0 & 1.0 & $\ldots^{\dagger}$ & $52 s$ & bad weather \\
\hline 071025 & 50033 & +0.0 & 0.4 & $17.3[84]$ & - & bad weather \\
\hline 071101 & 50038 & +1.0 & 10.6 & $>19.7[85]$ & $56 s$ & limit $[25]$ \\
\hline 071109 & 50040 & +0.0 & 1.0 & $>15.5[86]$ & $59 \mathrm{~s}$ & thick cirrus, limit [27] \\
\hline $071112 \mathrm{C}$ & 50044 & +0.4 & 11.0 & $17.5[87]$ & $64 \mathrm{~s}$ & bad weather \\
\hline 080320 & 50076 & +0.0 & 0.6 & $>20[88]$ & - & bad weather \\
\hline 080330 & 50079 & +0.0 & 1.2 & $16.8[89]$ & $400 \mathrm{~s}$ & manual, detection \\
\hline 080413A & 50082 & +0.0 & 1.6 & 15.0 & $60.7 \mathrm{~s}$ & detection [29] \\
\hline 080430 & 50090 & +0.8 & 7.4 & 17.5 & $34 \mathrm{~s}$ & detection $[31,32]$ \\
\hline 080517 & 50098 & +0.0 & 2.4 & $>18.5[90]$ & $3 \mathrm{~h} 22 \mathrm{~m}$ & weather delay \\
\hline 080603B & 50113 & +1.6 & 6.4 & $16.5[91]$ & $1 \mathrm{~h} 15 \mathrm{~m}$ & detection \\
\hline 080605 & 50115 & +0.0 & 3.8 & $17.9[92]$ & $43 \mathrm{~s}$ & detection [33] \\
\hline 080727C & - & +0.0 & 4.8 & $>19.9[93]$ & - & dome failure \\
\hline 080903 & - & +0.0 & 4.4 & $19.2[94]$ & - & dome failure \\
\hline 081001 & 50159 & +0.0 & 1.4 & - & - & bad weather \\
\hline 081003B & 50167 & +0.0 & 3.6 & $>17.6[35]$ & $41 \mathrm{~s}$ & OK, limit [35] \\
\hline 081126 & 50181 & +0.0 & 4.0 & $>18.0[95]$ & - & bad weather \\
\hline $081128 \mathrm{~A}$ & 50184 & +1.2 & 9.8 & $20.9[96]$ & - & bad weather \\
\hline 081210 & 50188 & +0.0 & 8.0 & $19.9[97]$ & $45 \mathrm{~m}$ & weather delay, out of focus \\
\hline 081228 & 50195 & +0.0 & 2.0 & $19.8[98]$ & - & bad weather \\
\hline 081230 & 50197 & +0.0 & 3.2 & $19.1[99]$ & - & bad weather \\
\hline
\end{tabular}

${ }^{\dagger}$ denotes discovered optical counterparts where there are not enough data to estimate the brightness $300 \mathrm{~s}$ after the GRB.

For example BOOTES-1B, which could detect mag $\sim 18$ at an unfiltered $60 \mathrm{~s}$ exposure, may detect an OT in about one third of the GRB triggers.

\section{Conclusions}

We have shown in a small historical retrospective the evolution of the telescope of BOOTES-1B, as it developed from the wide-field survey telescope to a dedicated GRB follow-up telescope.

Four years of BOOTES-1B GRB follow-up history are summarised for the first time transparently in the form of a table, which includes not only the observation status of BOOTES-1B, but also the time for which the object could have been observed, and a magnitude (or a limit) of the GRB optical afterglow 5 minutes after the trigger found in the literature. Every existing GRB trigger which was, or could 
have been observed by BOOTES-1B within 2 hours after the trigger is included. Twenty four successfully followed events are described case by case in a separate chapter. Many of these are published for the first time.

The data collected are also used to show the GRB trigger rate in Spain. By simply counting the triggers and the days during which they were collected, we estimate 18 triggers and about 50.5 hours of telescope time per year for real-time triggers. These numbers grow to about 22 triggers and 78.5 hours per year if we include also the GRBs observable within 2 hours after the trigger. We also derive the likelihood of the optical afterglow detection five minutes after the GRB trigger depending on the limiting magnitude of the telescope.

\section{Acknowledgments}

The Spanish side was supported by Spanish Ministry of Science and Technology by projects AYA 2004-01515, AYA 2007-63677, AYA2007-67627-C03-03, AYA2008-03467/ESP, and AYA2009-14000-C03-01. The Czech participation is supported by Grants of the Grant Agency of the Czech Republic 205/08/1207, 102/09/0997, and PECS 98023. Martin Jelínek was supported by the Ministry of Education and Science (MEC) Grant AP2003-1407.

\section{References}

[1] A. J. Castro-Tirado, J. Soldán, M. Bernas, et al., "The burst observer and optical transient exploring system (BOOTES)," Astronomy \& Astrophysics, vol. 138, pp. 583-585, 1999.

[2] A. J. Castro-Tirado, M. Jelínek, T. J. Mateo Sanguino, and A. de Ugarte Postigo, "BOOTES: a stereoscopic robotic ground support facility," Astronomische Nachrichten, vol. 325, no. 6-8, p. 679, 2004.

[3] P. Kubánek, M. Jelínek, S. Vítek, A. de Ugarte Postigo, M. Nekola, and J. French, "RTS2: a powerful robotic observatory manager," in Advanced Software and Control for Astronomy, vol. 6274 of Proceedings of SPIE, Orlando, Fla, USA, 2006.

[4] A. J. Castro-Tirado, J. M. Castro Cerón, J. Gorosabel, et al., "Detection of an optical transient following the 13 March 2000 short/hard gamma-ray burst," Astronomy \& Astrophysics, vol. 393, no. 3, pp. L55-L59, 2002.

[5] A. de Ugarte Postigo, "Antonio de Ugarte Postigo, Gammaray burst afterglows and instrumentation for their study: From BOOTES to X-shooter," Ph.D. thesis, Universidad de Granada, Granada, Spain, 2007.

[6] R. Quimby, E. McMahon, and J. Murphy, "GRBlog: a database for gamma-ray bursts," Astrophysics, vol. 727, pp. 529-532, 2003.

[7] M. Jelínek, et al., "GRB 050215b, BOOTES-1B optical limit," GRB Coordinates Network, Circular Service, 3023, 2005.

[8] M. Jelínek, et al., "Swift-BAT detection of GRB050505," GRB Coordinates Network, Circular Service, 3360, 2005.

[9] A. de Ugarte Postigo, M. Jelínek, and J. Gorosabel, "GRB 050505: early time observations," GRB Coordinates Network, Circular Service, 3376, 2005.

[10] A. J. Castro-Tirado, A. de Ugarte Postigo, J. Gorosabel, et al., "GRB 050509b: the elusive optical/nIR/mm afterglow of a short-duration GRB," Astronomy \& Astrophysics, vol. 439, pp. L15-L18, 2005.
[11] D. Band, J. Cummings, M. Perri, et al., "GRB 050525: Swift detection of a bright, possibly short burst," GRB Coordinates Network, Circular Service, 3466, 2005.

[12] S. T. Holland, "Swift-BAT detection of possible GRB 050528," GRB Coordinates Network, Circular Service, 3496, 2005.

[13] M. Jelínek, et al., "GRB050528: BOOTES optical candidate (retraction)," GRB Coordinates Network, Circular Service, 3500, 2005.

[14] A. J. Blustin, et al., "Swift/UVOT photometry of GRB050730," GRB Coordinates Network, Circular Service, 3717, 2005.

[15] S. A. Pandey, A. J. Castro-Tirado, S. McBreen, et al., "Multiwavelength afterglow observations of the high redshift GRB 050730," Astronomy \& Astrophysics, vol. 460, p. 415, 2006.

[16] S. Campana, S. Barthelmy, D. Burrows, et al., "GRB050824: Swift-BAT detection of a burst," GRB Coordinates Network, Circular Service, 3866, 2005.

[17] J. Sollerman, J. P. U. Fynbo, and J. Gorosabel, "The nature of the X-ray flash of August 24 2005," Astronomy \& Astrophysics, vol. 466, no. 3, pp. 839-846, 2007.

[18] J. B. Haislip, M. C. Nysewander, D. E. Reichart, et al., "A photometric redshift of $z=6.39 \pm 0.12$ for GRB 050904," Nature, vol. 440, no. 7081, pp. 181-183, 2006.

[19] M. Jelínek, et al., "GRB 051109a: BOOTES R \& I-band detection of the early afterglow," GRB Coordinates Network, Circular Service, 4227, 2005.

[20] M. Jelínek, et al., "GRB 051211A and GRB051211B: BOOTES simultaneous observations," GRB Coordinates Network, Circular Service, 4333, 2005.

[21] P. Boyd, et al., "Swift-BAT detection of a possible burst," GRB Coordinates Network, Circular Service, 4376, 2005.

[22] A. de Ugarte Postigo, et al., "GRB 051221B: BOOTES optical observations," GRB Coordinates Network, Circular Service, 4379, 2005.

[23] J. P. Halpern and S. Tyagi, "Possible GRB 051221B: MDM optical detection," GRB Coordinates Network, Circular Service, 4381, 2005.

[24] S. McBreen, et al., "GRB 071101: Swift detection of a burst," GRB Coordinates Network, Circular Service, 7030, 2007.

[25] M. Jelínek, et al., "GRB 071101: BOOTES-2 prompt optical observations," GRB Coordinates Network, Circular Service, 7032, 2007.

[26] S. Mereghetti, et al., "GRB 071109: a long GRB detected by INTEGRAL," GRB Coordinates Network, Circular Service, 7046, 2007.

[27] A. de Ugarte Postigo, et al., "GRB 071109: BOOTES-2 prompt optical observations," GRB Coordinates Network, Circular Service, 7047, 2007.

[28] J. Mao, et al., "GRB 080330: Swift detection of a burst with an optical counterpart," GRB Coordinates Network, Circular Service, 7537, 2008.

[29] P. Kubánek, et al., "GRB 080413A Bootes observation,” GRB Coordinates Network, Circular Service, 7603, 2008.

[30] M. Jelínek, et al., in preparation.

[31] M. Jelínek, et al., “GRB 080430: Bootes observation,” GRB Coordinates Network, Circular Service, 7648, 2008.

[32] A. de Ugarte Postigo, et al., in preparation.

[33] M. Jelínek, et al., "GRB080605: observation by BOOTES," GRB Coordinates Network, Circular Service, 7837, 2008.

[34] D. Gotz, et al., "GRB 081003B: a long GRB detected with INTEGRAL," GRB Coordinates Network, Circular Service, 8317, 2008. 
[35] M. Jelínek, et al., "GRB081003B: optical limits from Spain," GRB Coordinates Network, Circular Service, 8320, 2008.

[36] S. A. Yost, et al., "GRB050215B: ROTSE-III optical observations," GRB Coordinates Network, Circular Service, 3022, 2005.

[37] R. M. Quimby, et al., "GRB 050306: ROTSE-III early limits," GRB Coordinates Network, Circular Service, 3107, 2005.

[38] E. S. Rykoff, S. A. Yost, and H. Swan, "GRB 050421: ROTSE-III optical limits," GRB Coordinates Network, Circular Service, 3304, 2005.

[39] C. Guidorzi, A. Monfardini, A. Gomboc, et al., "The early multicolor afterglow of GRB 050502a: possible evidence for a uniform medium with density clumps," The Astrophysical Journal, vol. 630, pp. L121-L124, 2005.

[40] T. Poole, et al., “GRB050509a: Swift UVOT observations,” GRB Coordinates Network, Circular Service, 3394, 2005.

[41] P. Wozniak, et al., "GRB 050509b: RAPTOR deep, early limits," GRB Coordinates Network, Circular Service, 3414, 2005.

[42] A. J. Blustin, D. Band, S. Barthelmy, et al., "Swift panchromatic observations of the bright gamma-ray burst GRB 050525a," The Astrophysical Journal, vol. 637, pp. 901-913, 2006.

[43] S. D. Hunsberger, et al., "GRB050922C-Swift UVOT preliminary analysis," GRB Coordinates Network, Circular Service, 4041, 2005.

[44] C. G. Mundell, et al., "Faulkes Telescope R-band detection of GRB051111," GRB Coordinates Network, Circular Service, 4250, 2005.

[45] A. Klotz, et al., "GRB 051221B: TAROT optical limits,” GRB Coordinates Network, Circular Service, 4386, 2005.

[46] P. Roming, et al., "GRB 051227: Swift/UVOT upper limits (Possible U-band)," GRB Coordinates Network, Circular Service, 4411, 2005.

[47] A. Klotz, et al., "GRB 060111: TAROT optical observation," GRB Coordinates Network, Circular Service, 4483, 2006.

[48] A. Melandri, et al., "GRB 060204C: Liverpool Telescopes optical limit," GRB Coordinates Network, Circular Service, 4665, 2006.

[49] C. Guidorzi, et al., "GRB060206: Liverpool Telescope observation,” GRB Coordinates Network, Circular Service, 4693, 2006.

[50] A. Breeveld and A. Moretti, "GRB 060219: Swift/UVOT upper limits," GRB Coordinates Network, Circular Service, 4798, 2006.

[51] A. Melandri, et al., "GRB060319: Liverpool Telescope optical limit," GRB Coordinates Network, Circular Service, 4889, 2006.

[52] M. Jelínek, et al., "GRB 060418: FRAM early afterglow observation,” GRB Coordinates Network, Circular Service, 4976, 2006.

[53] V. Lipunov, et al., “GRB(?) 060421:MASTER optical limit," GRB Coordinates Network, Circular Service, 4988, 2006.

[54] T. S. Poole and V. La Parola, "GRB 060502A: Swift/UVOT source detection," GRB Coordinates Network, Circular Service, 5068, 2006.

[55] M. Jelínek, et al., “GRB 060507: BART limit," GRB Coordinates Network, Circular Service, 5092, 2006.

[56] M. D. Pasquale and J. Cummings, "Swift/UVOT observations of GRB060512,” GRB Coordinates Network, Circular Service, 5130,2006
[57] A. Klotz, M. Boer, and J. Atteia, "GRB 060515: TAROT optical observation,” GRB Coordinates Network, Circular Service, 5134, 2006.

[58] S. T. Holland, "GRB 060522: Swift/UVOT observations of the optical Afterglow," GRB Coordinates Network, Circular Service, 5158, 2006

[59] S. Vítek, et al., "GRB060602A: bootes-IR observations," GRB Coordinates Network, Circular Service, 5201, 2006.

[60] V. Lipunov, et al., "GRB060712: MASTER optical observation," GRB Coordinates Network, Circular Service, 5303, 2006.

[61] A. Klotz, M. Boer, and J. Atteia, "GRB 060814: TAROT optical observations," GRB Coordinates Network, Circular Service, 5448, 2006.

[62] A. Gomboc, "GRB060825: Liverpool Telescope optical observations," GRB Coordinates Network, Circular Service, 5476, 2006.

[63] A. Klotz, M. Boer, and J. Atteia, "GRB 060904: TAROT optical observations," GRB Coordinates Network, Circular Service, 5506, 2006.

[64] E. S. Rykoff, et al., "Looking into the Fireball: rotse-III and swift observations of early gamma-ray burst afterglows," The Astrophysical Journal, vol. 702, pp. 489-505, 2009.

[65] M. Jelínek, et al., in preparation.

[66] M. Nekola, et al., "GRB 060929: BART observation," GRB Coordinates Network, Circular Service, 5658, 2006.

[67] A. Klotz, et al., "GRB 061019: TAROT La Silla observatory optical observations," GRB Coordinates Network, Circular Service, 5731, 2006.

[68] H. Mikuz, et al., "GRB061110B: optical observations at Crni Vrh,” GRB Coordinates Network, Circular Service, 5808, 2006.

[69] A. Klotz, et al., "GRB 061217: TAROT Calern observatory optical observations," GRB Coordinates Network, Circular Service, 5942, 2006.

[70] P. D'Avanzo, et al., "GRB 061218: REM prompt observations," GRB Coordinates Network, Circular Service, 5937, 2006.

[71] A. de Ugarte Postigo, et al., "GRB 061222B: BOOTES-IR optical observations," GRB Coordinates Network, Circular Service, 5968, 2006.

[72] S. T. Holland and T. Sakamoto, "GRB 070103: Swift/UVOT upper limits," GRB Coordinates Network, Circular Service, 5993, 2006.

[73] M. M. Chester and O. Godet, "GRB 070129: Swift/UVOT upper limits," GRB Coordinates Network, Circular Service, 6066, 2007.

[74] A. Breeveld and T. Sakamoto, "GRB 070219: Swift/UVOT upper limits," GRB Coordinates Network, Circular Service, $6111,2007$.

[75] M. Stamatikos, et al., "GRB 070220: Swift detection of a burst," GRB Coordinates Network, Circular Service, 6114, 2007.

[76] W. Landsman, et al., "GRB070223: Swift/UVOT refined analysis," GRB Coordinates Network, Circular Service, 6134, 2007.

[77] S. T. Holland, et al., "GRB 070224: Swift/UVOT upper limits," GRB Coordinates Network, Circular Service, 6152, 2007.

[78] H. Mikuz, et al., "GRB 070411: optical photometry at Crni Vrh," GRB Coordinates Network, Circular Service, 6288, 2007.

[79] H. Mikuz and B. Dintinjana, "GRB 070412: optical observations at Crni Vrh,” GRB Coordinates Network, Circular Service, 6284, 2007.

[80] E. S. Rykoff, et al., "GRB 070429: ROTSE-III optical limits," GRB Coordinates Network, Circular Service, 6356, 2007.

[81] M. de Pasquale and C. B. Markwardt, "Swift/UVOT refined analysis of GRB070531," GRB Coordinates Network, Circular Service, 6481, 2007. 
[82] A. Stefanescu, et al., "GRB 070610: OPTIMA-Burst high-timeresolution optical observations," GRB Coordinates Network, Circular Service, 6492, 2007.

[83] A. Meshcheryakov, et al., "GRB 070704: RTT150 optical observations," GRB Coordinates Network, Circular Service, 6617, 2007.

[84] E. S. Rykoff, et al., "GRB 071025: ROTSE-III refined analysis," GRB Coordinates Network, Circular Service, 6992, 2007.

[85] M. Uemura, et al., "GRB071101: optical and NIR upper limits by KANATA," GRB Coordinates Network, Circular Service, 7037, 2007.

[86] A. Klotz, et al., “GRB 071109: TAROT Calern observatory optical observations," GRB Coordinates Network, Circular Service, 7052, 2007.

[87] B. Dintinjana, et al., "GRB 071112C: optical observations at Crni Vrh,” GRB Coordinates Network, Circular Service, 7078, 2007.

[88] G. G. Williams and P. A. Milne, "GRB 080320: early superLOTIS observations," GRB Coordinates Network, Circular Service, 7492, 2008.

[89] A. Klotz, et al., "GRB 080330: TAROT Calern observatory optical observations," GRB Coordinates Network, Circular Service, 7543, 2008.

[90] A. M. Parsons, et al., "GRB 080517: Swift detection of a burst," GRB Coordinates Network, Circular Service, 7742, 2008.

[91] N. P. M. Kuin, et al., "GRB 080603B: Swift/UVOT detection of the afterglow," GRB Coordinates Network, Circular Service, $7808,2008$.

[92] V. Rumyantsev, et al., "GRB080605: optical observations," GRB Coordinates Network, Circular Service, 7833, 2008.

[93] E. A. Hoversten and A. M. Parsons, "GRB 080727C: Swift/UVOT upper limits," GRB Coordinates Network, Circular Service, 8043, 2008.

[94] F. E. Marshall, et al., "GRB 080903: Swift/UVOT observations," GRB Coordinates Network, Circular Service, 8179, 2008.

[95] B. Gendre, et al., "GRB 081126: TAROT Calern observatory optical observations," GRB Coordinates Network, Circular Service, 8555, 2008.

[96] L. P. Xin, et al., "GRB 081128: optical observation of xinglong TNT," GRB Coordinates Network, Circular Service, 8572, 2008.

[97] S. Immler, et al., "GRB 081210: Swift/UVOT refined analysis," GRB Coordinates Network, Circular Service, 8654, 2008.

[98] P. Afonso, et al., "GRB 081228: GROND confirmation of the optical/NIR afterglow," GRB Coordinates Network, Circular Service, 8752, 2008.

[99] F. Yuan, et al., "GRB 081230: ROTSE-III detection of optical counterpart," GRB Coordinates Network, Circular Service, 8754, 2008. 

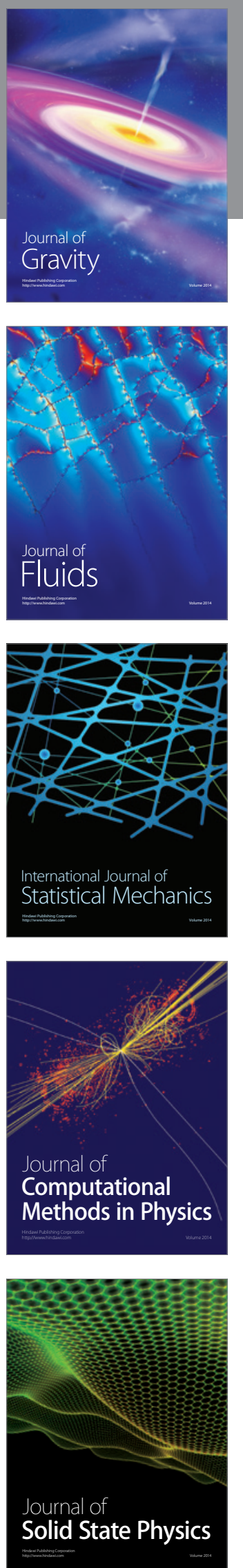

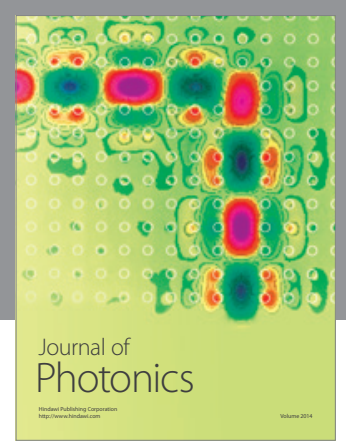

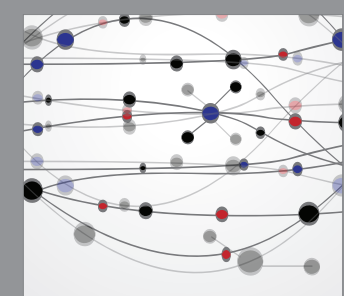

The Scientific World Journal
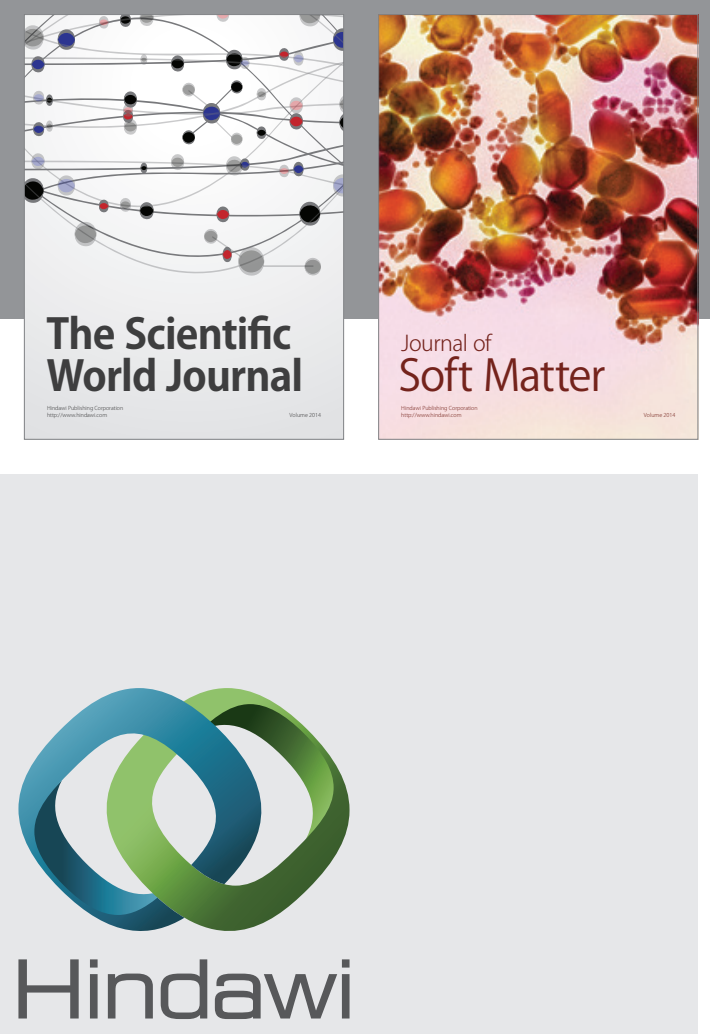

Submit your manuscripts at

http://www.hindawi.com
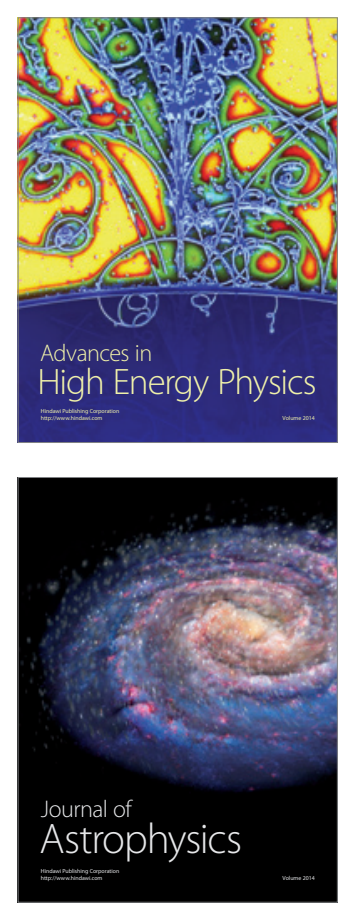
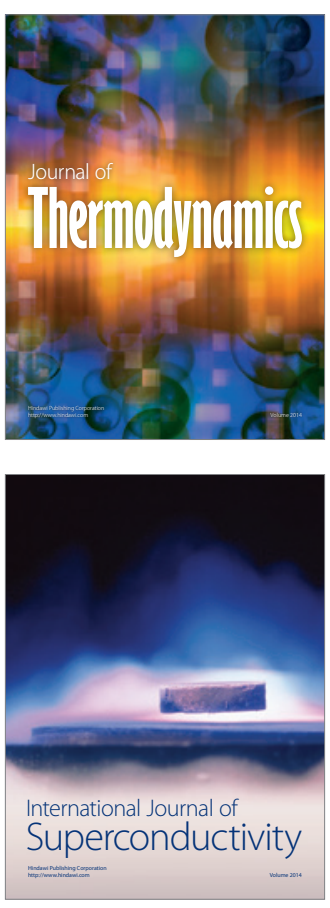
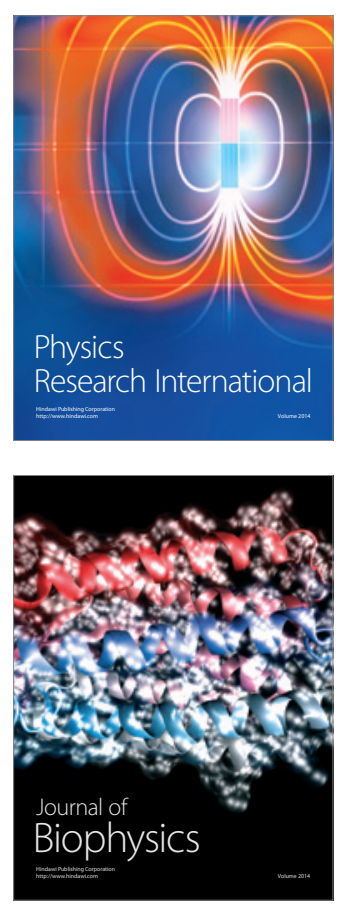
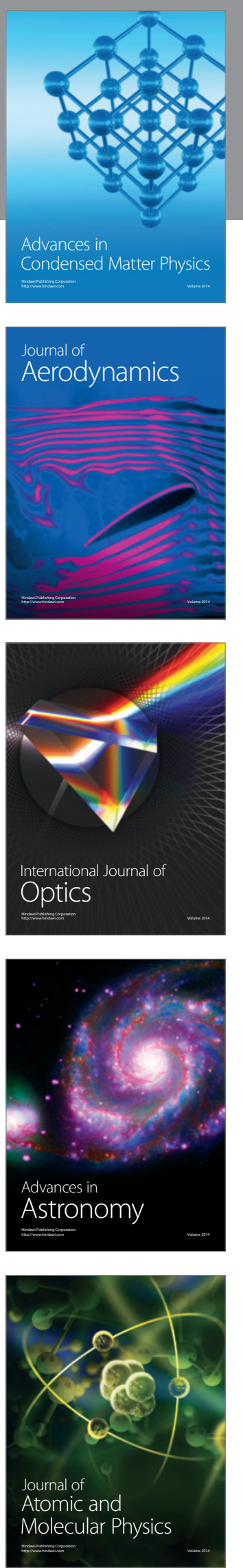